\title{
Professionalisierung inklusiver Schulentwicklungsprozesse
}

\section{Eine Analyse von Schulentwicklungszielen im Kontext einer Fortbildungsreihe für Lehrkräfte}

\section{Poldi Kuhl \& Ulrike Beate Müller}

Journal für Psychologie, 27(2), 192-211

https://doi.org/10.30820/0942-2285-2019-2-192

www.journal-fuer-psychologie.de

\section{Zusammenfassung}

Die Umsetzung schulischer Inklusion (UN-BRK 2008) stellt Schulen und Lehrkräfte vor große Herausforderungen, auf die Lehrkräfte im Rahmen ihres Studiums oft nicht hinreichend vorbereitet wurden (Werning 2017). Einem entsprechend großen Fortbildungsbedarf steht jedoch oft kein passendes Fortbildungsangebot gegenüber (Richter, Kuhl und Pant 2012). Die Fortbildungsreihe $\gg \operatorname{Jede}(\mathrm{r})$ ist besonders $«$ (JIB) der Deutschen Schulakademie (DSA) intendiert, zur Professionalisierung der schulischen Arbeit von Schulleitungen und Lehrkräften beizutragen und gute inklusive Praxis zu verbreiten. Die begleitende wissenschaftliche Evaluation, die JIB-Evaluation, soll es mithilfe einer Triangulation verschiedener methodischer Zugänge ermöglichen, evidenzbasiert Aussagen über die Wirksamkeit von JIB zu treffen. Im vorliegenden Beitrag werden erste Befunde der Analysen zu Schulentwicklungszielen, die sich die Schulen im Rahmen von JIB gesetzt haben, berichtet und diskutiert, um Transfer- und Implementationsprozesse im Kontext sich verändernder Schulen nachzuzeichnen.

Schlüsselwörter: Inklusive Schulentwicklung, Schulentwicklungsziele, Lehrkräfteprofessionalisierung, Umgang mit Heterogenität, Evaluation

\section{Summary}

Professionalization of Inclusive School Development Processes. An Analysis of School Development Goals in the Context of a Teacher Training Series

The implementation of inclusive schooling (UN-BRK 2008) poses major challenges for schools and teachers. Through their studies teachers often feel unprepared for working in inclusive schools and express a need for further training (Werning 2017). This need, however, is often not matched by suitable training possibilities (Richter, Kuhl, and Pant 2012). The teacher training series »Everybody is Unique « (Jede $[\mathrm{r}]$ ist besonders - JIB), developed by the German School Academy (Deutsche Schulakademie - DSA) intends to contribute to 
the professionalization of principals and teachers and to disseminate good inclusive practice. The accompanying scientific evaluation of JIB aims to provide evidence on the effectiveness of JIB by triangulating various methodological approaches. In this paper, we present first findings on the school development goals JIB participants reported for their schools. Moreover, we discuss these school development goals as means of developmental processes in the context of changing schools.

Key words: inclusive school development, school development goals, teacher professionalization, dealing with heterogeneity, evaluation

\section{$1 \quad$ Einleitung}

Die Umsetzung schulischer Inklusion im Zuge der Ratifizierung der UN-Behindertenrechtskonvention (UN-BRK 2008) stellt Schulen und Lehrkräfte auf verschiedenen Ebenen, wie beispielsweise der Unterrichtsgestaltung, der Zusammenarbeit im (multiprofessionellen) Kollegium und der Ausgestaltung außerschulischer Kooperationen, vor große Herausforderungen. Viele Lehrkräfte werden durch die Inklusion von Kindern mit sonderpädagogischem Förderbedarf (SPF) mit Anforderungen konfrontiert, auf die sie im Rahmen ihres Lehramtsstudiums nur selten vorbereitet wurden (Werning 2017). Dem entsprechend großen Fortbildungsbedarf in den Themenfeldern sonderpädagogische Förderung, Umgang mit Heterogenität und Individualisierung steht jedoch oft kein passendes Fortbildungsangebot gegenüber (Richter, Kuhl und Pant 2012).

Eine Bestandsaufnahme zu inklusionsbezogenen Fortbildungsmaßnahmen verdeutlicht, dass diese oft von kurzer Dauer sind, selten wissenschaftlich evaluiert und häufig nur von einer einzelnen Lehrkraft einer Schule besucht werden (Amrhein und Badstieber 2013). Eine erfolgreiche inklusive Schulentwicklung zeichnet sich allerdings durch die Veränderung der Schule als Mehrebenen-System sowohl auf der institutionellen, didaktischen, interpersonellen als auch auf der professionellen Handlungsebene aus (Prengel 2013). Schulische Veränderungen werden maßgeblich von der kollegialen Unterstützung des Transfers von in Fortbildungen erworbenen Kompetenzen bestimmt (Burke und Hutchins 2007). Für den erfolgreichen Transfer in die Schule als günstig erweist es sich, dass nach aktuellen Studien Lehrkräfte Inklusion an sich mehrheitlich befürworten und sie vielfach selbst auch schon über Erfahrung im Umgang mit Kindern mit sonderpädagogischem Förderbedarf verfügen (Forsa 2017).

Effektive Fortbildungen zeichnen sich unter anderem dadurch aus, dass sie es Lehrkräften über einen längeren Zeitraum ermöglichen, auf die eigene Berufspraxis bezogene Herausforderungen zu bearbeiten und zu reflektieren (Lipowsky 2010). Dieses Element der stetigen Arbeit an schulspezifischen Herausforderungen auf der Grundlage 
von Schulentwicklungszielen ist Teil des Konzeptes der Fortbildungsreihe »Jede(r) ist besonders « (JIB). Schwerpunkt des vorliegenden Beitrags ist die Analyse der Schulentwicklungsziele, die sich die Teilnehmenden im Rahmen von JIB gesetzt haben. Im Kern soll mit diesem Beitrag der Frage nachgegangen werden, welche Veränderungen an den Teilnehmerschulen parallel zur Teilnahme an JIB initiiert wurden. Genauer betrachtet wird, welche Ziele sich Schulen setzten, wie sie die Zielerreichungsprozesse in den Schulen implementierten und welche Aspekte sie dabei als unterstützend oder herausfordernd erlebten.

\section{Theoretischer Hintergrund und Forschungsstand}

\subsection{Inklusion und Umgang mit Heterogenität}

Im deutschen Bildungssystem ist in den letzten Jahren eine stärkere Heterogenität der Schülerschaft festzustellen, die auf verschiedene Entwicklungen zurückgeht. Sowohl der strukturelle gesellschaftliche Wandel der Schülerschaft in sozialer, kultureller und sprachlicher Hinsicht als auch Veränderungen durch Schulreformen, vor allem die Umsetzung der gemeinsamen Beschulung von Kindern mit und ohne SPF auf Grundlage der Ratifizierung der UN-BRK, sind für eine wachsende Heterogenität der Schüler/innen mitbestimmend (Budde 2017).

Einem weiten Inklusionsverständnis folgend, beziehen sich aktuelle Inklusionsbestrebungen nicht ausschließlich auf Schüler/innen mit SPF, sondern zielen vielmehr darauf ab, Schulen so zu gestalten, dass sie allen Kindern - unabhängig von individuellen Merkmalen wie beispielsweise Geschlecht und Herkunft - ein Umfeld bieten, in dem sie sich gut entwickeln können (Faulstich-Wieland 2015). Ein solches, weites Verständnis von Inklusion (Hinz 2013), das im Kern darauf abzielt, Kinder nicht in passende Schulen zu selektieren, sondern Schulen vielmehr derart zu öffnen, dass sie allen Kindern passende Entwicklungsmöglichkeiten bieten, liegt auch der gemeinsamen Empfehlung für eine »Lehrerbildung für eine Schule der Vielfalt « zugrunde (KMK und HRK 2015).

Auch wenn damit die Zielstellung für schulische Entwicklungsprozesse gesetzt zu sein scheint und der gemeinsame Unterricht aller Kinder mit und ohne Behinderung grundsätzlich von der Mehrheit der Pädagog/innen befürwortet wird (Forsa 2017), wird die derzeitige Umsetzung in den Schulen durchaus kritisch eingeschätzt (Rjosk et al. 2017). Dies zeigen zum Beispiel auch die Ergebnisse einer aktuellen ForsaUmfrage (2019), in der etwa ein Viertel der befragten Schulleiter/innen angab, Inklusion/Integration derzeitig als zweitgrößtes Problem an der Schule zu sehen. Fast alle befragten Schulleiter/innen gaben zudem an, dass sich aus dem Umgang mit neuen 
Herausforderungen, wie Inklusion, für die meisten Lehrkräfte an der Schule Mehrbelastungen ergeben. Demnach sind Inklusion und der Umgang mit Heterogenität aktuell bedeutsame Anliegen, auf die im Rahmen von Schulentwicklungsprozessen Lehrkräftefortbildungen mit Angeboten reagieren sollten (Amrhein und Badstieber 2013).

\subsection{Beitrag von Fortbildung zur Entwicklung professioneller Handlungskompetenzen von Lehrkräften im Umgang mit Heterogenität}

Fortbildungen als Maßnahmen der Professionalisierung von Lehrkräften werden nicht nur, aber insbesondere in den Bereichen relevant, für die Lehrkräfte im Studium nicht hinreichend vorbereitet wurden. Inklusion zählt unter anderem zu den aktuell relevanten Bereichen, in denen über Fortbildungen zum berufsbegleitenden Kompetenzerwerb von Lehrkräften beigetragen werden soll (Werning 2012).

Fortbildungen, die wirksam sind und entsprechend bis in die Schule hineinwirken, zeichnen sich laut Darling-Hammond et al. (2009) dadurch aus, dass sie einen fachlichen Fokus aufweisen, sich an Merkmalen lernwirksamen Unterrichts orientieren und wissenschaftliche Expertise heranziehen. Zudem werden in wirksamen Fortbildungen Phasen von Input, Erprobung und Reflexion miteinander verschränkt, die Möglichkeit zu Rückmeldungen bieten, sodass sich auch Gelegenheiten zum Erleben der eigenen Wirksamkeit ergeben. Wie Amrhein und Badstieber (2013) in ihrer Expertise jedoch verdeutlichten, weisen inklusionsbezogene Fortbildungen diese Merkmale in der Regel nicht auf und werden zudem bislang nur selten wissenschaftlich evaluiert.

Eine aktuelle Ausnahme hiervon bilden die begleitenden Evaluationen zweier Fortbildungen zur inklusiven Schulentwicklung für die beteiligten Lehrkräfte. Jäntsch et al. (2015) nahmen im Rahmen des Pilotprojekts Inklusive Grundschulen (PING) die Kompetenzzuwächse von Lehrkräften vor und nach der Fortbildungsreihe in den Blick und komplettierten ihre Auswertungen mit Daten aus einer Interviewstudie. Schuck, Rauer und Prinz (2018) kamen mit der Studie Evaluation der Einführung der inklusiven Bildung in Hamburgs Schulen (EiBiSCH) zu ähnlichen Befunden, wenn beide Arbeitsgruppen berichten, dass die begleitende Fortbildung nicht allen schulischen Bedarfen gerecht wurde. Hierdurch wurde die Notwendigkeit der Ausrichtung der Angebote an persönlichen und schulischen Voraussetzungen für den potenziellen Erfolg von Professionalisierungsmaßnahmen deutlich. Moser (2018) schreibt zudem Lehrkräften und ihren eigenen Überzeugungen und Werthaltungen zum Umgang mit Heterogenität eine zentrale Rolle zu. Im Rahmen der Aus- und Fortbildung von Lehrkräften käme es insbesondere darauf an, Gelegenheiten zu schaffen, damit (angehende) Lehrkräfte ihr Wissen, ihre Kompetenzen, aber auch Einstellungen reflexiv verknüpfen können. 
Da Inklusion aber nicht alleinig dadurch erreicht wird, dass einzelne Lehrkräfte ihren eigenen Unterricht verändern, sondern vielmehr eine Aufgabe darstellt, die Schulen auf allen Ebenen und im Hinblick auf alle im schulischen Kontext tätigen Professionen betrifft (Werning 2013), bedarf es auch im Rahmen von Fortbildungen einer stärkeren Orientierung an und Konzentration auf (inklusive) Schulentwicklungsprozesse, die im Folgenden kurz erörtert werden.

\subsection{Inklusive Schulentwicklung}

Im Bereich der Schulentwicklungsforschung in Deutschland ist das Drei-Wege-Modell der Schulentwicklung zentral, das postuliert, dass Schulentwicklungsprozesse in den Bereichen Organisations-, Personal- und Unterrichtsentwicklung ablaufen (Rolff 1998; 2016). Organisationsentwicklung als Weiterentwicklung von Organisationen verläuft entlang drei aufeinanderfolgender Phasen des Organisationswandels von der Initiation über die Implementation hin zur Inkorporation und damit zur Institutionalisierung. Organisationsentwicklung kann sich nach Rolff (2016) auf unterschiedlichste Bereiche von Schule beziehen, unter anderem Schulprogramm, Schulkultur, Erziehungsklima, Schulmanagement, Teamentwicklung, Evaluation, Kooperation und Steuergruppe. Für die Personalentwicklung sind nach Rolff (2016) Komponenten der Personalfortbildung, der Personalführung, der Personalförderung und der (personalbezogenen) Persönlichkeitsentwicklung von Bedeutung, die beispielsweise durch Kommunikationstraining, Schulleitungsberatung, Hospitationen, Jahresgespräche, Zielvereinbarungen und Führungs-Feedback in Schulen wirksam werden. Unterricht und seine Entwicklung sieht Rolff (2016) schließlich an zentraler Position in der Schule und folglich auch in Maßnahmen der Lehrkräfteprofessionalisierung. Unterrichtsentwicklung erfolgt nach Rolff (2016) beispielsweise durch Maßnahmen in den Bereichen Fachlernen, überfachliches Lernen, Methodentraining, Selbstlernfähigkeit, Schülerorientierung, Öffnung, erweiterte Unterrichtsformen und Lernkultur. Alle drei Bereiche der Schulentwicklung stehen im Drei-Wege-Modell in einem Systemzusammenhang, sodass jeder der drei Wege notwendigerweise auch zu den zwei anderen Wegen führt und diese sich demnach wechselseitig beeinflussen.

Neben diesem allgemeinen und etablierten Modell zur Schulentwicklung von Rolff $(1998$; 2016) finden sich in den letzten Jahren auch zunehmend Modelle und Theorien der Schulentwicklung, die sich spezifisch auf inklusive Schulen und deren Veränderungsprozesse beziehen. Schulen, die sich erfolgreich im Hinblick auf Inklusion entwickeln, kennzeichnen sich durch Veränderungen in drei zentralen Bereichen (Werning 2013): Sie entwickeln schulintern ein gemeinsames Verständnis der geteilten Werte, verbessern die Kooperation im (multiprofessionellen) Kollegium und bauen ih- 
re außerschulischen Netzwerke und Kooperationen aus. In ähnlicher Weise finden sich diese zentralen Gelingensbedingungen für inklusive Schulentwicklung, die Inklusion zu einem gesamtschulischen Vorhaben machen, auch in den anderen Ansätzen inklusiver Schulentwicklung wie dem Inklusionsindex von Boban und Hinz (2014), dem Mehrebenenmodell inklusiver Schulentwicklung (Heimlich 2003) oder dem Modell inklusiver Schulentwicklung, das Arndt und Werning (2016) auf Grundlage der Analyse von Jakob-Muth-Preisträgerschulen vorlegten.

Während mit diesen Modellen bereits eine Reihe von theoretischen Ansätzen zur inklusiven Schulentwicklung vorliegen, wird der Forschungsstand in diesem Bereich als bislang unzureichend und wenig belastbar bezeichnet (Preuss-Lausitz 2014). Die kürzlich erschienene EiBiSCH-Studie (Schuck, Rauer und Prinz 2018) verdeutlichte, dass sich die Prozesse und Bedarfe zwischen den Schulen in Abhängigkeit der Schulart, der Erfahrung mit inklusiv/integrativer Arbeit und dem Sozialraum, in dem sich die Schulen befanden, unterschieden. Damit wurde bekräftigt, dass Entwicklungsprozesse von Schulen entlang der Ausgangslagen und Rahmenbedingungen vor allem einzelschulisch ausgestaltet werden (Moser 2017).

\section{Untersuchung von Schulentwicklungszielen im Rahmen der JIB-Fortbildung}

\subsection{Forschungsinteresse und Fragestellungen}

Mit den vorliegenden theoretischen Annahmen sowohl im Bereich der inklusiven Lehrkräfteprofessionalisierung als auch im Bereich inklusiver Schulentwicklung liegt umfangreiches Wissen über potenzielle Gelingensbedingungen für Inklusion vor. Weniger gut untersucht ist bislang hingegen, welche Schulentwicklungsziele Schulen anstoßen, um dem Ziel schulischer Inklusion näher zu kommen. Müller (2012) wies darauf hin, dass schulische Entwicklungsprozesse durch Zielsetzungen initiiert oder begleitet werden können und verdeutlichte die Notwendigkeit, Zielsetzungen innerhalb von Schule zwischen Akteursgruppen abzustimmen und allen Beteiligten transparent zu machen.

Die Formulierung von Schulentwicklungszielen, wie sie im Rahmen der vorliegenden Studie »Jede(r) ist besonders « (JIB) formuliert wurden, ermöglicht es den Teilnehmer/innen, die in der Fortbildung thematisierten Inhalte im Hinblick auf die eigene Schule und die eigenen berufsbezogenen Herausforderungen zu reflektieren. Eine solche intensive reflexive Auseinandersetzung mit der Ausgangslage und den Entwicklungsprozessen der eigenen Schule wurde als ein Merkmal wirksamer Lehrkräftefortbildungen identifiziert (Darling-Hammond et al. 2009; Lipowsky 2010). Als Kernstück dieses Beitrags wird auf Grundlage der folgenden Fragestellungen unter- 
sucht, welche Veränderungen an den Teilnehmerschulen parallel zur Teilnahme an JIB initiiert wurden:

1. Welche Schulentwicklungsziele setzen sich die teilnehmenden Schulen im Rahmen der JIB-Fortbildungsreihe?

2. Wie werden die Schulentwicklungsziele in den einzelnen Schulen umgesetzt und welche Aspekte sind den Schulen bei der Umsetzung bedeutsam?

3. Welche Aspekte werden im Hinblick auf die Erreichung der Schulentwicklungsziele als unterstützend und welche als herausfordernd erlebt?

\subsection{Forschungskontext}

\subsubsection{Die Fortbildungsreihe »Jede(r) ist besonders« (JIB)}

Die Fortbildungsreihe »Jede(r) ist besonders « (JIB) wurde von der Deutschen Schulakademie (DSA) konzipiert, um zur Professionalisierung der schulischen Arbeit von Schulleitungen und Lehrkräften beizutragen. Das Konzept zu JIB wurde in Orientierung an den sechs Qualitätsbereichen des Deutschen Schulpreises entwickelt (Beutel, Höhmann, Pant und Schratz 2016). Damit wurde im Rahmen von JIB nicht nur der Unterricht in den Blick genommen, sondern durch Berücksichtigung von Aspekten wie Schulentwicklungsprozessen, Kooperation und Schulleben auch verdeutlicht, dass inklusive Schulentwicklung ein komplexer Prozess ist, der die ganze Schule auf mehreren Ebenen und unter Beteiligung verschiedener Akteursgruppen umfasst. Entsprechend wurden neben den unterrichtsbezogenen Themengebieten Leistung und Unterricht auch die Themenfelder Schulentwicklung, Vernetzung und Schulleben in der JIB-Fortbildungsreihe aufgegriffen und jeweils praktisch auf die Möglichkeiten und Herausforderungen inklusiver Schulentwicklung an den Teilnehmerschulen bezogen.

Basierend auf einem Train-the-Trainer-Konzept wurden zunächst fünfTrainerteams aus Schulpreisträgerschulen an ihren eigenen Schulen zum Umgang mit Heterogenität geschult. Zudem wurden sie dafür qualifiziert, im Anschluss an die eigene Teilnahme an JIB, in der Heimatregion JIB mit fünf Schulen durchzuführen. An den fünf regionalen Zirkeln nahmen je fünf Schulen mit jeweils drei Teilnehmer/innen (mind. eine Person aus der Schulleitung) teil, sodass aus 25 Schulen insgesamt 75 Teilnehmende an JIB beteiligt waren (siehe 3.3 für die genauere Stichprobenbeschreibung).

Über einen Gesamtzeitraum von zwei Jahren wurden acht JIB-Module zweitägig in Form von Werkstätten an Fortbildungsstätten in den fünf Regionen ausgerichtet. Bei der inhaltlichen Ausgestaltung von JIB wurde zum einen das Ziel verfolgt, zur Haltungsänderung von Schulleitungen und Lehrkräften im Kontext inklusiver Schule beizutragen. Zum anderen sollte die Fortbildungsreihe die Teilnehmenden dabei unterstützen, in 
ihren eigenen Schulen der Ausgangslage und den Bedürfnissen entsprechende Schulentwicklungsziele zu formulieren und geeignete Entwicklungsprozesse zu initiieren.

\subsubsection{Die JIB-Evaluation als wissenschaftliche Begleitevaluation von JIB}

Die Durchführung von JIB an den 25 Schulen in den fünf Regionen wird begleitet durch eine wissenschaftliche Begleitevaluation, die JIB-Evaluation. Diese verfolgt die folgenden Ziele: (1) die Wirksamkeit der JIB-Fortbildungsmaßnahme für Schulentwicklungsprozesse an den teilnehmenden Schulen empirisch zu überprüfen sowie (2) die Schulentwicklungsprozesse an diesen Schulen nachzuzeichnen und so Gelingensbedingungen und auch Barrieren erfolgreicher Schulentwicklung für den Umgang mit Heterogenität abzuleiten.

Um diesen Zielen nachgehen zu können, wird im Rahmen der wissenschaftlichen Begleitevaluation ein längsschnittlicher Mixed-Methods-Ansatz verfolgt. Dabei werden verschiedene qualitative und quantitative Forschungsmethoden herangezogen, unter anderem Fragebogenerhebungen, Interviews und Beobachtungen. Eine Triangulation der verschiedenen methodischen Zugänge im Rahmen der wissenschaftlichen Begleitevaluation soll es ermöglichen, evidenzbasiert Aussagen über die Wirksamkeit von JIB zu treffen. Der vorliegende Beitrag geht auf das Design der JIB-Evaluation nicht konkreter ein, sondern konzentriert sich ausschließlich auf die Auswertungen der schulspezifischen Entwicklungsziele, die Schulen parallel zur Teilnahme an JIB initiierten.

\subsection{Stichprobe}

Die qualifizierten JIB-Trainer/innen veröffentlichten in ihren Heimatregionen Ausschreibungen, um interessierte Schulen für die Teilnahme an JIB zu gewinnen. Die so gewonnenen 25 Teilnehmerschulen aus den fünf Regionen entstammten unterschiedlichen Schularten. So nahmen insgesamt acht Grundschulen, vier reine Sekundarstufe ISchulen, drei Schulen mit Grundschule und Sekundarstufe I, sechs Schulen mit Sekundarstufe I und II, eine stufenübergreifende Schule von Klasse 1 bis 13, zwei Berufsschulen und eine Förderschule an JIB und damit auch an der JIB-Evaluation teil.

Auch hinsichtlich der geografischen Lage unterschieden sich die JIB-Schulen. Vier Schulen befinden sich im ländlichen Raum, sieben Schulen in einer Kleinstadt, eine Schule befindet sich in einem Vorort einer Großstadt und 13 schließlich in einer Großstadt. Eine Schule hatte insgesamt weniger als 100 Schüler/innen, 14 Schulen zwischen 100 und 500 Schüler/innen, sechs Schulen zwischen 500 und 1000 Schüler/innen und vier Schulen schließlich mehr als 1000 Schüler/innen.

Hinsichtlich der Erfahrung der Schulen bezüglich des Unterrichtens von Kindern mit SPF wurden zwischen den teilnehmenden Schulen Unterschiede deutlich. Bis auf 
eine Schule gaben alle JIB-Schulen an, Kinder mit SPF zu unterrichten und wiesen hierbei unterschiedlich viel Erfahrung auf. Gruppiert man die Schulen anhand einer Terzilbildung, fallen Schulen mit bis zu sieben Jahren gemeinsamen Unterrichts in die Gruppe der Schulen mit keiner oder wenig Erfahrung. Schulen mit mittlerer Erfahrung weisen entsprechend integrativ/inklusive Praxen seit acht bis 24 Jahren auf. Schulen im höchsten Terzil schließlich sind die im gemeinsamen Unterricht erfahrensten und berichten diesbezügliche Praktiken von mehr als 25 Jahren.

Alle 25 Schulen wurden um Angaben zu ihren Schulentwicklungszielen im Kontext von JIB gebeten, wobei keine Angabe darüber erfolgte, wer aus dem JIB-Schulteam den Fragebogen ausgefüllt hatte. Rückantworten zu den Schulentwicklungszielen trafen von 23 der 25 Schulen ein, sodass die entsprechende Antwortquote bei 92\% lag. Eine Schule hatte vor dem Befragungszeitpunkt bereits mit der Zirkelleitung entschieden, nicht weiter an JIB teilzunehmen. Eine weitere Schule schickte den Fragebogen auch nach mehrfacher Erinnerung ohne Angabe von Gründen nicht zurück und konnte so nicht für die Auswertung der Schulentwicklungsziele berücksichtigt werden.

\subsection{Erhebungsinstrumente}

Der Fragebogen zur Erfassung der Schulentwicklungsziele wurde für die Erhebung neu entwickelt. Die JIB-Teams der Teilnehmerschulen wurden darin gebeten, ihre maximal drei wichtigsten Schulentwicklungsziele kurz zu beschreiben, die sie sich für ihre Schule im Zusammenhang mit der JIB-Fortbildung gesetzt haben. Pro Ziel gaben die Schulen in einer kurzen Beschreibung in einem offenen Format an, wie dieses in der Schule umgesetzt, der Prozess der Zielerreichung begleitet und ausgewertet wird. Darüber hinaus wurden Herausforderungen und als hilfreich empfundene Aspekte bei der Zielerreichung erfragt. Abschließend wurden Schulen zielübergreifend um Auskunft dazu gebeten, wie wichtig ihnen verschiedene Aspekte bei der Erreichung der Schulentwicklungsziele waren. Zudem bewerteten sie, welche übergeordneten Ziele mit der Umsetzung der spezifischen JIB-Ziele an der Schule erreicht werden sollten.

\section{Ergebnisse}

\subsection{Welche Schulentwicklungsziele setzten sich die teilnehmenden Schulen im Rahmen von JIB?}

15 der 23 Schulen, die an der Befragung teilnahmen, benannten drei Ziele. Fünf Schulen gaben zwei Ziele an und drei Schulen genau ein Ziel. Einer Schule war das Benennen 
eines vierten Ziels sehr wichtig, sodass diese Schule auch ein viertes Ziel angab. Insgesamt wurden von den 23 teilnehmenden Schulen folglich 59 Schulentwicklungsziele benannt.

Zur Analyse der Schulentwicklungsziele wurde die strukturierende Inhaltsanalyse nach Mayring (2015) herangezogen, um diese zu kategorisieren. Als deduktive Hauptkategorien dienten mit der Unterscheidung nach Organisations-, Personal- und Unterrichtsentwicklung (OE, PE, UE) die Hauptwege der Schulentwicklung nach Rolff (2016). Jedes genannte Ziel wurde zunächst mithilfe der strukturierenden Inhaltsanalyse (Mayring 2015) basierend auf der Benennung des Ziels und der genaueren Kurzbeschreibung einer dieser drei Oberkategorien zugeordnet. Die Kategorisierung aufEbene der drei Oberkategorien wurde unabhängig von zwei Personen vorgenommen und erzielte eine hohe Interraterübereinstimmung von 0,95. Dabei wurde ersichtlich, dass knapp die Hälfte aller genannten Ziele (29 von 59; 49,1\%) dem Ziel der Organisationsentwicklung gewidmet waren. Fast ebenso häufig wurden Ziele benannt, die den Entwicklungsschwerpunkt auf die Unterrichtsentwicklung legten (23 von 59; $39,0 \%)$. Ziele zur Personalentwicklung hingegen spielten eine vergleichsweise geringe Rolle (7 von 59; 11,9\%).

Um die Schulentwicklungsziele nicht nur abstrakt auf die Oberkategorien zu beziehen, sondern auch detaillierter in Beziehung zu den Bedingungen auf Einzelschulebene setzen zu können, wurden sie ferner hinsichtlich der Schulart unterschieden. Aufgrund der geringen Anzahl wurden Förder- und Berufsschulen sowie Schulen mit kombinierter Grund- und Sekundarstufe hierbei nicht gesondert berücksichtigt. Es zeigte sich, dass sich die Schulentwicklungsziele der acht Grundschulen überwiegend und recht ausgeglichen auf Unterrichtsentwicklung und Organisationsentwicklung bezogen (OE: 50\%, UE: 45\%, PE: 5\%). Die insgesamt sechs Schulen mit Sekundarstufe I und II konzentrierten sich in ihren Zielsetzungen am häufigsten auf Unterrichtsentwicklung, etwas weniger, aber ebenfalls häufig auf Organisationsentwicklung und in vergleichsweise geringem Ausmaß auf Personalentwicklung (UE: 50\%, OE: 37,5\%, PE: 12,5\%). Die reinen Sekundarstufe I-Schulen setzen ihren Schwerpunkt sehr deutlich bei der Unterrichtsentwicklung, seltener bei der Organisationsentwicklung und formulierten keine Ziele bezüglich der Personalentwicklung (UE: 71,4\%, OE: 28,6\%, PE: $0 \%)$.

Zusätzlich zu Schulartunterschieden wurde auch untersucht, inwiefern sich die Zielsetzungen der Schulen in Abhängigkeit ihrer Erfahrungen im Unterrichten von Kindern mit SPF unterschieden. Abbildung 1 verdeutlicht die unterschiedlichen Entwicklungsschwerpunkte in den Schulen mit wenig, mittlerer und viel Erfahrung im Unterrichten von Kindern mit SPF. Hierbei wird entlang der Erfahrung mit inklusiv/integrativer Arbeit ersichtlich, dass sich Schulen, die bislang über keine oder wenig Erfahrung im Unterrichten von Kindern mit SPF verfügen, in stärkerem Maße Ziele 
auf der Ebene der Unterrichtsentwicklung formulierten. Schulen, die bereits über mehr Erfahrung verfügten, benannten hingegen häufiger Ziele auf der Ebene der Organisations- oder Personalentwicklung (Abbildung 1).

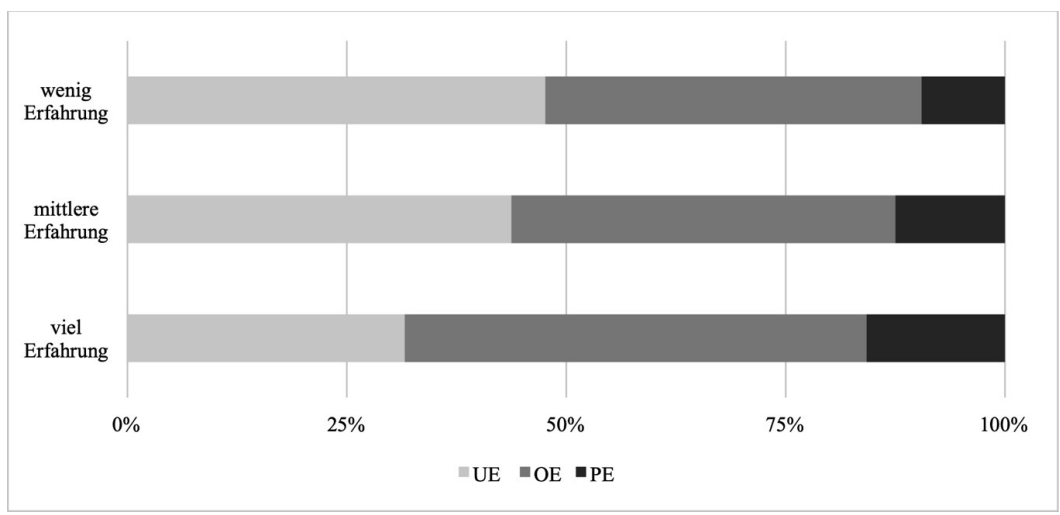

Abbildung 1: Kategorisierung der Schulentwicklungsziele nach den drei Wegen der Schulentwicklung nach Rolff (2016) in Unterrichtsentwicklung (UE), Organisationsentwicklung (OE), Personalentwicklung (PE) sowie der Erfahrung der Schule mit dem Unterrichten von Schüler/innen mit sonderpädagogischem Förderbedarf (wenig [0-7 Jahre]; mittel [7-24 Jahre], viel [mehr als 25 Jahre])

Zieht man neben der Erfahrung im Unterrichten der Kinder mit SPF auch die Schularten heran, so wird deutlich, dass es vor allem die Grundschulen sind, die bereits über mittlere oder viel Erfahrung verfügen (7 von 8, 87,5\%). Der Anteil der Schulen mit mittlerer oder viel Erfahrung unter den Sekundarschulen hingegen lag bei 40\% (4 von $10)$.

Zur vertieften inhaltlichen Erschließung erfolgte in einem weiteren Schritt die Zuordnung jedes Schulentwicklungsziels zu einer Unterkategorie der drei Oberkategorien OE, UE und PE. Hierzu wurde ein detaillierteres Kategoriensystem auf Grundlage des Drei-Wege-Modells der Schulentwicklung zugrunde gelegt (Rolff 2016), das induktiv erweitert wurde, wenn Ziele benannt wurden, die zwar klar einer Oberkategorie zuzuordnen waren, aber nicht unter den von Rolff (2016) benannten Subkategorien verortet werden konnten. Auch die Kategorisierung auf der Ebene der Unterkategorien (Tabelle 1) erfolgte durch zwei Personen zunächst unabhängig voneinander, war aber insgesamt weniger eindeutig im Vergleich zur Bestimmung der Oberkategorien (Interraterübereinstimmung: 0,83). Unklare Zuordnungen wurden daher zusätzlich von einer dritten Person kodiert und die Kategorisierung schließlich konsensuell bestimmt. 


\begin{tabular}{lll}
\hline $\begin{array}{l}\text { Organisationsentwicklung } \\
\text { (29 Ziele) }\end{array}$ & $\begin{array}{l}\text { Unterrichtsentwicklung } \\
\text { (23 Ziele) }\end{array}$ & $\begin{array}{l}\text { Personalentwicklung } \\
\text { (7 Ziele) }\end{array}$ \\
\hline - Teamentwicklung (24,2\%) & - Schülerorientierung (43,5\%) & $\begin{array}{l}\text { - Päd. Umgang mit speziellen } \\
\text { Schüler/innengruppen (42,8\%) }\end{array}$ \\
- Schulmanagement (20,7\%) & - Lernkultur (17,4\%) & - Kommunikationstraining (14,3\%) \\
- Kooperation (17,2\%) & - Selbstlernfähigkeit (13,0\%) & - Lehrer-Feedback (14,3\%) \\
- Schulkultur (13,8\%) & - Fachlernen (8,7\%) & - Supervision/Coaching (14,3\%) \\
- Steuergruppe (10,3\%) & - Erweiterte Unterrichtsformen & - (personalbezogene) \\
& $(8,7 \%)$ & $\begin{array}{l}\text { Persönlichkeitsentwicklung } \\
(14,3 \%)\end{array}$ \\
& & \\
- Schulprogramm (10,3\%) & - Überfachliches Lernen (4,4\%) & \\
- Erziehungsklima (3,5\%) & - Methodentraining (4,3\%) & \\
\hline
\end{tabular}

Tabelle 1: Zuordnung der Schulentwicklungsziele zu Haupt- und Unterkategorien (in Anlehnung an Rolff 2016)

Hinsichtlich der Unterrichtsentwicklung wurden am häufigsten Schulentwicklungsziele angegeben, die Schülerorientierung ins Zentrum der Bemühungen der Schulen rückten. Ein Beispiel hierfür ist das Ziel »Umgang mit Heterogenität«, das die betreffende Schule näher bezeichnete als » Förderung jedes Einzelnen nach seinen Möglichkeiten, Methoden der Binnendifferenzierung gezielt einsetzen «. Bezogen auf die Organisationsentwicklung bilden die recht ähnlichen Subkategorien Teamentwicklung und Kooperation den größten Themenbereich. Beispielhaft sei hier das Ziel »Teams weiterentwickeln « einer Schule genannt, die in der Beschreibung des Ziels angab, dass »Bestehende Teams an der Schule mit aus dem JIB-Projekt erlernten Werkzengen optimiert werden sollen. Auch die Routine ist ein Ziel, das Erleichterung schafft «. Im Bereich der Personalentwicklung konzentrierten sich die Ziele vor allem auf die Bereiche Pädagogischer Umgang mit speziellen Schüler/innengruppen und Kommunikationstrainings, zielten aber auch auf Lehrer-Feedback, Supervision/Coaching und (personalbezogene) Persönlichkeitsentwicklung ab. Beispielhaft benannte eine Schule ihr Ziel im Bereich Supervision/Coaching als »Unterstützungssysteme für Kollegen ohne sonderpädagogische Expertise « und beschrieb es näher als »Etablierung verbindlicher Strukturen zur Fallberatung und praktischen Unterstützungen der Kollegen v. a. in der Klassenleitung «.

Bezogen auf den Zeitraum der Schulentwicklungszielumsetzung haben die Schulen zu jedem von ihnen formuliertem Ziel angegeben, wann sie dieses erreicht haben wollen. Für die Auswertung wurden die Erreichungszeiträume nach kurzfristig (null bis sechs Monate), mittelfristig (ein bis zwei Jahre) und langfristig (drei bis fünf Jahre) unterschieden. Im Ergebnis zeigte sich, dass die meisten Ziele für einen mittelfristigen Zeitraum (27 von 59, 45,8\%) oder einen kurzfristigen Zeitraum (17 von 59, 28,8\%) 
geplant wurden. Einige Ziele wurden für einen langfristigen Zeitraum geplant (sieben von 59, 11,9\%), für einen Teil der Ziele wurde jedoch auch kein Zeitraum angegeben (acht von 59, 13,6\%).

Ein weiterer, für die Untersuchung inklusiver Schulentwicklung relevanter Aspekt ist die Frage danach, ob die formulierten Schulentwicklungsziele der JIB-Schulen einen expliziten Inklusionsbezug aufwiesen. Folglich wurden alle Ziele im Hinblick auf ihren Inklusionsbezug ausgewertet. Als explizit-inklusive Schulentwicklungsziele wurden die Ziele klassifiziert, die inklusionsbezogene Begriffe, wie zum Beispiel »Inklusion «, »Integration « oder »Umgang mit Vielfalt bzw. Heterogenität « wörtlich enthielten (Beispiel: Entwicklung eines inklusiven Schulcurriculums). Dies traf auf 20,3\% der Ziele zu (12 von 59). Der Großteil der Ziele ( 47 von 59, 79,7\%,) wies zwar keinen expliziten Inklusionsbezug auf, genannte Ziele wie beispielsweise »Alternative Leistungsmessung« oder »Optimierung des Arbeitens im Team « könnten in Schulen aber auch indirekt langfristig zu einer erfolgreichen inklusiven Schulentwicklung beitragen. Da der Inklusionsbezug jedoch nicht explizit geäußert wurde und somit der Interpretation durch das Evaluationsteam unterliegt, wurden diese Ziele als nicht-explizit inklusiv kategorisiert. Betrachtet man die Ziele zusätzlich zum Inklusionsbezug auch zur Oberkategorie (OE, PE und UE), so wird deutlich, dass von den zwölf Schulentwicklungszielen mit explizitem Inklusionsbezug die meisten in den Bereich der Unterrichtsentwicklung (8 von 12, 66,7\%), einige in den Bereich der Organisationsentwicklung ( 4 von 12,33,3\%) und kein Ziel in den Bereich der Personalentwicklung fiel.

\subsection{Wie wurden die angestrebten Schulentwicklungsziele in den einzelnen Schulen umgesetzt und begleitet und welche Aspekte sind den Schulen bei der Umsetzung wichtig?}

Gefragt nach der Art und Weise, wie die Schulen ihre Schulentwicklungsziele umsetzten und begleiteten, schilderten die JIB-Teams unterschiedliche Vorgehensweisen. Knapp die Hälfte der genannten Schulentwicklungsprozesse wurden in den Schulen durch verschiedene Formen des kollegialen Austauschs begleitet (z. B. Teamgespräche: 47,5\%). Für etwa ein Viertel der Schulentwicklungsziele wurden Fortschritte systematisch dokumentiert (z. B. Protokolle: 25,4\%). Darüber hinaus wurden von den Schulen auch Formen der Evaluation (z. B. Reflexion und Feedback: 15,5\%) sowie externe Kooperationen (z. B. Zusammenarbeit mit Universitäten: 15,5\%) als Monitoringmaßnahmen zu den Schulentwicklungsprozessen benannt.

Zielübergreifend bewerteten die JIB-Teams des Weiteren die Wichtigkeit verschiedener Aspekte, die mit der Umsetzung der Schulentwicklungsziele an den Schulen erreicht werden sollten im Format einer vierstufigen Likert-Skala von 1 (»trifft gar 
nicht $\mathrm{zu} \ll$ ) bis 4 (» trifft voll zu «). Dabei wurde deutlich, dass den JIB-Schulen während der Umsetzung insbesondere die Aspekte gute Zusammenarbeit mit den Kolleg/innen $(M=3,90, S D=0,31)$, die Berücksichtigung verschiedener Perspektiven $(M=3,85$, $S D=0,49)$, klar aufgeteilte Verantwortlichkeiten $(M=3,75, S D=0,48)$ und verständlich formulierte Ziele $(M=3,69, S D=0,50)$ wichtig waren. Als für die Zielerreichung insgesamt weniger relevant wurden hingegen bezüglich der Erreichung der Schulentwicklungsziele der transparente Austausch mit den Eltern $(M=3,08, S D=1,04)$ und die gemeinsame Zielsetzung mit den Schüler/innen eingeschätzt $(M=2,78, S D=1,04)$. Während die erstgenannten Ziele allen Schulen ähnlich wichtig erschienen, zeigen sich bei den letztgenannten Zielen Schulartunterschiede. Der Austausch mit den Eltern war vor allem den Grundschulen ein Anliegen $\left(M_{\mathrm{GS}}=3,37, S D_{\mathrm{GS}}=0,92 ; M_{\mathrm{SEK}}=2,50\right.$, $S D_{\text {SEK }}=1,31$ ), die gemeinsame Zielsetzung mit den Schüler/innen hingegen wurde an den Sekundarschulen als wichtiger erachtet $\left(M_{\mathrm{GS}}=2,25, S D_{\mathrm{GS}}=1,16 ; M_{\mathrm{SEK}}=2,75\right.$, $\left.S D_{\text {SEK }}=1,16\right)$.

Die Schulen bewerteten zudem zielübergreifend einige Fragen dazu, was sie mit der Umsetzung ihrer Entwicklungsziele an der Schule erreichen wollen. Im Format einer vierstufigen Likert-Skala von 1 (»trifft gar nicht zu «) bis 4 ( $\gg$ trifft voll zu «) bewerteten sie eine Reihe von vorgegebenen Zielstellungen, die sich unter anderem an den Qualitätsbereichen des Deutschen Schulpreises orientierten (Beutel, Höhmann, Pant und Schratz 2016). Die drei dabei wichtigsten Ziele betrafen den besseren Umgang mit Verschiedenheit $(M=3,90, S D=0,31)$, die Verbesserung der Zusammenarbeit in der Schule $(M=3,86, S D=0,47)$ und eine positivere Haltung zum Umgang mit Vielfalt im Kollegium $(M=3,85, S D=0,36)$. Als vergleichsweise weniger bedeutsam wurden hingegen die Aspekte Beitrag zur Selbstevaluation in Schule und Unterricht $(M=2,51, S D=0,94)$ sowie Verbesserung der außerschulischen Kooperationen $(M=2,42, S D=1,04)$ bewertet.

\subsection{Welche Aspekte wurden im Hinblick auf die Erreichung der Schulentwicklungsziele als unterstützend und herausfordernd erlebt?}

Im Hinblick auf die Evaluation des Beitrags von JIB ist von besonderem Interesse, welche Merkmale des Fortbildungsangebotes als unterstützend erlebt wurden. Folglich wurden die JIB-Schulteams bezogen auf jedes Schulentwicklungsziel darum gebeten, im Format einer vierstufigen Likert-Skala von 1 (»trifft gar nicht $\mathrm{zu} \ll$ ) bis 4 (»trifft voll $\mathrm{zu} \ll)$ zu beurteilen, wer oder was sie bei der Umsetzung des jeweiligen Ziels unterstützt. Über alle Ziele hinweg wurden insbesondere Teambesprechungen als unterstützend erlebt $(M=3,49, S D=0,78)$. Im Hinblick auf die Schulentwicklungsprozesse wurden zudem an zweiter und dritter Stelle Wissen und Fähigkeiten aus dem JIB-Zirkel $(M=3,44, S D=0,63)$ genannt. Als ebenfalls unterstützend, aber etwas weniger 
bedeutsam wurde die Unterstützung durch neu eingerichtete Steuergruppen $(M=3,15$, $S D=1,05)$, die JIB-Zirkelleitungen $(M=2,69, S D=1,03)$ und externe Beratung/Supervision $(M=2,69, S D=1,20)$ eingeschätzt.

Als herausfordernd im Zusammenhang mit der schulischen Umsetzung ihrer Ziele beschrieben die Schulen insbesondere den Umgang mit dem Kollegium sowie mangelnde Ressourcen. Die genannten Herausforderungen zum Kollegium (30 von 59; 50,8\%) bezogen sich auf die Zusammenarbeit (11 von 30, 36,6\%), die Beachtung individueller Perspektiven (14 von 30, 46,7\%; z. B. zu Altersunterschieden oder verschiedenen Meinungen) und Widerstände im Kollegium (5 von 30, 16,7\%). Herausforderungen hinsichtlich mangelnder Ressourcen wurden für etwa ein Viertel der Ziele (11 von 59 $18,6 \%)$ geschildert. Diese bezogen sich vor allem auf erschwerte Bedingungen durch zeitliche, räumliche oder finanzielle Engpässe.

\section{Diskussion und Ausblick}

\subsection{Zusammenfassung und Diskussion}

Ziel des Beitrags war es zu untersuchen, welche Veränderungen an Schulen parallel zur Teilnahme an einer Lehrkräfteprofessionalisierungsmaßnahme für inklusive Schulentwicklung initiiert werden. Genauer in den Blick genommen wurde dabei, in welchen Bereichen Schulen Ziele spezifizieren, wie sie die Zielerreichungsprozesse implementieren und welche Aspekte dabei als unterstützend oder herausfordernd erlebt wurden.

Zunächst lässt sich resümierend auf die erste Fragestellung nach den Inhalten der Schulentwicklungsziele der JIB-Schulen festhalten, dass diese bei Zugrundelegung des Schulentwicklungsmodells nach Rolff (2016) ihre Schwerpunkte primär auf Entwicklungsprozesse im Bereich der Organisations- und Unterrichtsentwicklung legten, explizite Personalentwicklungsziele hingegen eine untergeordnete Rolle spielten. Den zusammengenommen größten Teil der Organisationsentwicklungsziele bildeten Ziele zur Teamentwicklung bzw. Kooperation. Diese Fokussierung auf kooperatives Lehrerhandeln schließt an etablierte Modelle inklusiver Schulentwicklung an, die diesen Entwicklungsbereich auch als bedeutsame Gelingensbedingung für Inklusion identifiziert haben (Arndt und Werning 2016; Boban und Hinz 2014; Heimlich 2003). Ebenfalls in Einklang mit diesen Modellen steht der Befund, dass im Bereich Unterrichtsentwicklung am häufigsten Schulentwicklungsziele angegeben wurden, die auf Schülerorientierung fokussierten. Bezüglich der Personalentwicklung wurden vor allem Ziele formuliert, die zur Kompetenzentwicklung von Lehrkräften in verschiedenen Bereichen beitragen sollten, zum Beispiel durch (Kommunikations-)Trainings, Maßnahmen zur (personalbezogenen) Persönlichkeitsentwicklung, Supervision/Coaching 
oder Lehrer-Feedback. Kompetenzen sowohl im Bereich des pädagogischen Umgangs mit Schüler/innen als auch der Kommunikation werden dem Bereich der kommunikativen Kompetenzen zugeordnet, die eine zentrale Kernkompetenz von Lehrkräften im Bereich der inklusiven Bildung darstellt (Fischer, Rott und Veber 2015).

Zusätzlich zur Analyse der inhaltlichen Schwerpunktsetzungen der Schulen wurde untersucht, ob sich diese in Abhängigkeit der Erfahrung der JIB-Schulen mit inklusiv/integrativer Arbeit unterschieden. Im Einklang mit Befunden von Schuck, Rauer und Prinz (2018) konzentrierten sich Schulen, die bislang über keine oder wenig Erfahrung im Unterrichten von Kindern mit SPF verfügten, in stärkerem Maße auf die Unterrichtsentwicklung. Dieser Befund untermauert die Argumentation von Heimlich (2003), dass Schulen, die mit inklusiver Arbeit beginnen, meist zunächst Entwicklungen auf der Unterrichtsebene anstoßen. Schulen, die bereits über längere Erfahrung mit inklusiv/integrativer Arbeit verfügten, könnten demnach in Bezug auf den Unterricht schon weiter fortgeschritten sein und daher vermehrt Ziele auf der Ebene der Organisations- oder Personalentwicklung benennen. Die gefundenen Schulartunterschiede nach den Schwerpunktsetzungen nach OE, UE und PE liegen möglicherweise in der unterschiedlichen Erfahrung der Schulen im gemeinsamen Unterricht begründet. Insbesondere Grundschulen zeichneten sich durch bereits umfangreiche Jahre Erfahrung aus und waren es, die ihren Fokus stärker auf Organisations- als auf Unterrichtsentwicklung legten. Die weiterführenden Schulen hingegen hatten mehrheitlich weniger Erfahrung und konzentrierten sich in stärkerem Maße auf die Unterrichtsentwicklung. Rauer, Schuck und Prinz (2018) legen entsprechend nahe, solche Unterschiede zu berücksichtigen und Fortbildungen stärker an den Ausgangslagen und Rahmenbedingungen von Schulen auszurichten.

Die zweite Fragestellung zur Untersuchung der Schulentwicklungsziele adressierte die Umsetzung an den Schulen. Im Zuge der Umsetzung wurden eine gute Kooperation im Kollegium und ein strukturierter Umsetzungsprozess als besonders bedeutsam herausgehoben. Die weitere Verbesserung der Zusammenarbeit in der Schule war den JIB-Schulen im Zuge der Umsetzung ihrer Ziele wichtig. Ähnliche Befunde finden sich auch bei Arndt und Werning (2016), die eine enge Kooperation im Kollegium und verbindliche Strukturen und Absprachen als Merkmale guter inklusiver Schulen identifizierten. Unterschiede zwischen Schularten wurden bei einzelnen Aspekten deutlich. So legten Grundschulen mehr Wert auf einen transparenten Austausch mit den Eltern, während an den Sekundarschulen die gemeinsame Zielsetzung mit den Schüler/innen bedeutsam war.

In der dritten Fragestellung der Auswertung wurden unterstützende und herausfordernde Aspekte erfragt. Als wichtigste Unterstützung wurden dabei Teambesprechungen genannt, während Steuergruppen als deutlich weniger hilfreich angegeben wurden. Dieser Befund geht einher mit Befunden von Voß (2013), die ebenfalls herausfand, dass 
die Einbindung aller professionellen Fachkräfte an inklusiven Schulen im Hinblick auf die inklusive Schulentwicklung produktiver war als die Einrichtung von Steuergruppen. An zweiter und dritter Stelle der bedeutsamen unterstützenden Elemente bei der Zielumsetzung wurden das Wissen und Fähigkeiten aus dem JIB-Zirkel angegeben, sodass die Fortbildung einen entscheidenden Anteil zur (inklusiven) Schulentwicklung beiträgt.

Das Kollegium wurde nicht nur als bedeutsamste Hilfe, sondern auch am häufigsten in Bezug auf Herausforderungen bei der Zielumsetzung genannt. Die Verständigung auf gemeinsame Werte und Visionen sowie die Verbesserung der Kooperationen in der Schule gehören entsprechend zu den wichtigsten Entwicklungsbereichen (Werning 2013), um zu erreichen, dass Inklusion und der Umgang mit Vielfalt als ein Anliegen verstanden wird, dass die ganze Schule betrifft (Arndt und Werning 2016).

\subsection{Limitationen und Ausblick}

Durch die Konzeption der Fortbildung, die es Schulen unter anderem ermöglicht, die eigenen inklusiven Schulentwicklungsprozesse über einen längeren Zeitraum mit vielfältigen Möglichkeiten zur Reflexion zu bearbeiten, weist JIB Merkmale auf, die in der Forschung als bedeutsam identifiziert wurden (Darling-Hammond et al. 2009; Lipowsky 2010). Ob sich diese langfristig auch in erfolgreichen Entwicklungen an den JIBSchulen zeigen werden, lässt sich aufgrund der hier ausschließlich berichteten Befunde zu den Schulentwicklungszielen nicht beurteilen. Die besondere Rolle, die Ziele für gelingende Schulentwicklungsprozesse spielen könnten, stellte auch Müller (2012) heraus. Die in diesem Beitrag analysierten Schulentwicklungsziele bilden nur einen Baustein der wissenschaftlichen Begleitevaluation. In Zusammenschau mit den Befunden der anderen Teiluntersuchungen sollen abschließend Gelingensbedingungen bzw. Barrieren erfolgreicher Schulentwicklung für den Umgang mit Heterogenität abgeleitet werden.

Limitierend muss zudem ergänzt werden, dass sich die bisherigen Befunde möglicherweise nicht ohne Weiteres auf Schulen im Allgemeinen übertragen lassen. Die Teilnahme an JIB erfolgte freiwillig und erforderte von den Schulen die grundsätzliche Bereitschaft, sich im Hinblick auf den Umgang mit Vielfalt verbessern und entsprechende personale Ressourcen einbringen zu wollen und zu können. Dies könnte insofern zu einer positiven Selektion der JIB-Schulen geführt haben, als dass sich Schulen beteiligten, die bereits für sich die Notwendigkeit von Veränderungen erkannt hatten und prinzipiell zur Teilnahme bereit waren.

Die Ergebnisse zu JIB im Allgemeinen und zu den Schulentwicklungsprozessen und deren Implementation in den Einzelschulen im Besonderen können zur Diskussion darüber beitragen, wie Lehrkräfte im Sinne lebenslangen Lernens auch berufsbegleitend für 
den Umgang mit Herausforderungen professionalisiert werden können. In künftigen Forschungsvorhaben bieten sich Potenziale insbesondere hinsichtlich der Evaluation bis auf die Ebene der Lernergebnisse von Schüler/innen und auch bezüglich der Fragen, wie für gelingende inklusive Schulentwicklung zentrale Kompetenzen und auch Haltungen bereits im Lehramtsstudium thematisiert und vermittelt werden können (Moser 2018).

\section{Literatur}

Amrhein, Bettina, und Benjamin Badstieber. 2013. Lehrerfortbildungen zu Inklusion - eine Trendanalyse. Gütersloh: Bertelsmann-Stiftung. http://www.bertelsmann-stiftung.de/cps/rde/xbcr/SID -DE66D7BB-B75F0088/bst/xcms_bst_dms_38700_38701_2.pdf.

Arndt, Ann-Kathrin, und Rolf Werning. 2016. Was kann man von Jakob-Muth-Preisträgerschulen lernen? Ergebnisse der Studie »Gute inklusive Schule«. In Inklusion kann gelingen! Forschungsergebnisse und Beispiele guter schulischer Praxis, Bertelsmann Stiftung, (Hrsg.), 105-140. Gütersloh: Verlag Bertelsmann-Stiftung.

Beutel, Silivia-Iris, Höhmann, Katrin, Pant, Hans A. und Michael Schratz. 2016. Handbuch Gute Schule. Sechs Qualitätsbereiche für eine zukunftsweisende Praxis. Leipzig: Friedrich.

Boban, Ines, und Andreas Hinz. 2014. Der Index für Inklusion. Eine Hilfe für die inklusive Entwicklung der inklusiver werdenden Schule. Lernende Schule, H. 67, 12-15.

Budde, Jürgen. 2017. Heterogenität: Entstehung, Begriff, Abgrenzung. In Studienbuch Heterogenität, Bohl, Thorsten, Budde, Jürgen und Marcus Rieger-Ladich, (Hrsg.), 13-26. Bad Heilbrunn: utb.

Burke, Lisa A., und Holly M. Hutchins. 2007. Training transfer: An integrative literature review. Human resource development review, 6 (3), 263-296.

Darling-Hammond, Linda, Wei, Ruth C., Andree, Alethea, Richardson, Nikole und Stelios Orphanos. 2009. Professional learning in the learning profession: A status report on teacher development in the United States and abroad. National Staff Development Council and the School Redesign Network at Stanford University.

Faulstich-Wieland, Hannelore. 2015. Umgang mit Heterogenität und Differenz. In Sexuelle Vielfalt im Handlungsfeld Schule. Konzepte aus Erziehungswissenschaft und Fachdidaktik, Huch, Sarah und Martin Lücke, (Hrsg.), 49-67. Bielefeld: transcript.

Fischer, Christian, David Rott und Marcel Veber. 2015. Kompetenzorientierte Lehrer/innenbildung durch Individuelle Schüler/innenförderung. In: Umgang mit Vielfalt. Aufgaben und Herausforderungen für die Lehrerinnen- und Lehrerbildung. Fischer, Christina, Veber, Marcel, FischerOntrup, Christiane, und Rafael Buschmann, (Hrsg.), 77-100. Münster und New York: Waxmann.

Forsa Politik- und Sozialforschung. 2017. Inklusion an Schulen aus Sicht der Lehrkräfte in Deutschland - Meinungen, Einstellungen und Erfahrungen. Ergebnisse einer repräsentativen Lehrerbefragung. Umfrage im Auftrag des VBE. Berlin. https://www.vbe.de/fileadmin/user_uplo-ad/ VBE/Service/Meinungsumfragen/2017_05_10_Inklusion_an_Schulen_Auswertung.pdf

Forsa Politik-und Sozialforschung. 2019.DieSchule aus Sicht derSchulleiterinnen und Schulleiter-Berufszufriedenheitvon Schulleitungen. Ergebnisseeinerbundesweiten Repräsentativbefragung. Umfrage im Auftrag des VBE. Berlin. https://www.vbe.de/fileadmin/user_upload/VBE/Veranstaltungen/ Deutscher_Schulleiterkongress/2019/2019-03-15_forsa-Bericht_Schulleitungen_Bund.pdf Heimlich, Ulrich. 2003. Integrative Pädagogik. Eine Einführung. Stuttgart: Kohlhammer. 
Hinz, Andreas. 2013. »Inklusion - von der Unkenntnis zur Unkenntlichkeit!? - Kritische Anmerkungen zu einem Jahrzehnt Diskurs über schulische Inklusion in Deutschland«. Zeitschrift für Inklusion. https://www.inklusion-online.net/index.php/inklusion-online/article/view/26/26

Jäntsch, Christian, Jessica Jaeuthe, Stefanie Bosse und Nadine Spörer. 2015. Fortbildung und Beratung zum inklusiven Unterricht. In Inklusives Lehren und Lernen im Land Brandenburg. Abschlussbericht zur Begleitforschung des Pilotprojektes »/nklusive Grundschule», Spörer, Nadine, Vock, Miriam, Schründer-Lenzen, Agi und Kai Maaz, (Hrsg.), 155-180. Ludwigsfelde: LISUM.

KMK und HRK (Sekretariat der Ständigen Konferenz der Kultusminister der Länder in der Bundesrepublik Deutschland; Hochschulrektorenkonferenz). 2015. Lehrerbildung für eine Schule der Vielfalt. Gemeinsame Empfehlung von Hochschulrektorenkonferenz und Kultusministerkonferenz. Beschluss der Kultusministerkonferenz vom 12.03.2015. Beschluss der Hochschulrektorenkonferenz vom 18.03.2015. https://www.kmk.org/fileadmin/veroeffentlichungen_ beschluesse/2015/2015_03_12-Schule-der-Vielfalt.pdf

Lipowsky, Frank. 2010. Lernen im Beruf. Empirische Befunde zur Wirksamkeit von Lehrerfortbildung«. In Lehrerinnen und Lehrer lernen. Konzepte und Befunde zur Lehrerfortbildung, Müller, Florian H, Eichenberger, Astrid, Lüders, Manfred und Johannes Mayr, (Hrsg.), 51-72. Münster: Waxmann.

Mayring, Philipp. 2015. Qualitative Inhaltsanalyse. Grundlagen und Techniken. 12., überarbeitete Auflage. Weinheim und Basel: Beltz.

Moser, Vera. 2017. Inklusion und Organisationsentwicklung. In Inklusion und Schulentwicklung, Moser, Vera und Marina Eggert, (Hrsg.), 15-30. Stuttgart: W. Kohlhammer.

Moser, Vera. 2018. Lehrerinnen- und Lehrerbildung. In Handbuch schulische Inklusion. Sturm, Tanja, und Monika Wagner-Willi, (Hrsg.), 283-298. Opladen und Toronto: Barbara Budrich.

Müller, Sabine. 2012. Ziele klären. In Handbuch Schulentwicklung und Schulentwicklungsberatung, Buhre, Claus G. und Hans-Günter Rolff, (Hrsg.), 109-115. Weinheim und Basel: Beltz Verlag.

Müller, Ulrike Beate, und Poldi Kuhl. 2019. Zur Bedeutung der Selbstwirksamkeit für eine individuelle Förderung im Unterricht mit heterogenen Lerngruppen. In Individuelle Förderung im Unterricht und in der Schule, Knauder, Hannelore, und Monika Reisinger, (Hrsg.), 39-50. Münster: Waxmann.

Prengel, Annedore. 2013. Inklusive Bildung in der Primarstufe. Eine wissenschaftliche Expertise des Grundschulverbandes. Unter Mitarbeit von E. Horn. Grundschulverband e. V.: Frankfurt/M.

Preuss-Lausitz, Ulf. 2014. WissenschaftlicheBegleitungen derWegezurinklusiven Schulentwicklung in den Bundes/ändern. Versuch einer Übersicht. Berlin: Autor. https://www.ewi.tu-berlin.de/fileadmin/ i49/dokumente/Preuss-Lausitz/Wiss._Begleitung_Inklusion.pdf (Zugriff am 28.08.2018).

Richter, Dirk, Poldi Kuhl und Hans A. Pant. 2012. Soziale Disparitäten. In Kompetenzen von Schülerinnen und Schülern am Ende der vierten Jahrgangsstufe in den Fächern Deutsch und Mathematik. Ergebnisse des IQB-Ländervergleichs 2011, Stanat, Petra, Pant, Hans A, Böhme, Katrin, und Dirk Richter, (Hrsg.), 191-208. Münster: Waxmann.

Rjosk, Camilla, Lars Hoffmann, Dirk Richter, Alexandra Marx und Cornelia Gresch. 2017. Qualifikation von Lehrkräften und Einschätzungen zum gemeinsamen Unterricht von Kindern mit und Kindern ohne sonderpädagogischen Förderbedarf. In IQB-Bildungstrend 2016. Kompetenzen in den Fächern Deutsch und Mathematik am Ende der 4. Jahrgangsstufe im zweiten Ländervergleich, Stanat, Petra, Schipolowski, Stefan, Rjosk, Camilla, Weirich, Sebastian, und Nicole Haag (Hrsg.), 335-353. Münster: Waxmann.

Rolff, Hans-Günther. 1998. Entwicklung von Einzelschulen. Viel Praxis, wenig Theorie und kaum Forschung - Ein Versuch, Schulentwicklung zu systematisieren. In: Jahrbuch der Schulentwicklung, Rolff, Hans-Günther, Bauer, Karl-Oswald., Klemm, Klaus, und Hermann Pfeiffer, (Hrsg.), 295-326. Band 10. Weinheim: Juventa. 
Rolff, Hans-Günther. 2016. Schulentwicklung kompakt. Modelle, Instrumente, Perspektiven. 3. Auflage. Weinheim: Beltz.

Schuck, Karl D., Wulf Rauer und Doren Prinz. 2018. EiBiSch - Evaluation inklusiver Bildung in Hamburgs Schulen. Quantitative und qualitative Ergebnisse. Münster und New York: Waxmann.

UN-BRK (UN-Behindertenrechtskonvention). 2008. Gesetz zu dem Übereinkommen der Vereinten Nationen vom 13. Dezember 2006 über die Rechte von Menschen mit Behinderungen vom 21. 12. 2008., §2.

Voß, Lena. 2013. Die Konzepte »Kompetenzzentren für sonderpädagogische Förderung« und »Regionale Integrationskonzepte« als Wegbegleiter für Inklusion. Eine qualitative Untersuchung. In Inklusion: Kooperation und Unterricht entwickeln, Werning, Rolf, und Ann-Kathrin Arndt, (Hrsg.), 63-85. Bad Heilbrunn: Klinkhardt.

Werning, Rolf. 2012. Inklusive Schulentwicklung. In Die inklusive Schule. Standards für die Umsetzung, Moser, Vera, (Hrsg.), 49-61. Stuttgart: Kohlhammer.

Werning, Rolf. 2013. Inklusive Pädagogik - Eine Herausforderung für die Schulentwicklung. In Besser gemeinsam lernen! Inklusive Schulentwicklung, Klauß, Theo, und Karin Terfloth, (Hrsg.), 17-28. Heidelberg: Winter.

Werning, Rolf. 2017. Aktuelle Trends inklusiver Schulentwicklung in Deutschland. Grundlagen, Rahmenbedingungen und Entwicklungsperspektiven. In Inklusion: Profile für die Schul- und Unterrichtsentwicklung in Deutschland, Österreich und der Schweiz. Theoretische Grundlagen - Empirische Befunde - Praxisbeispiele, Lütje-Klose, Birgit, Miller, Susanne, Schwab, Susanne, und Bettina Streese, (Hrsg.), 17-30. Münster: Waxmann.

\section{Die AutorInnen}

Poldi Kuhl, Prof. Dr. Dipl-Psych., ist Juniorprofessorin für Bildungswissenschaft, insbesondere Evaluation und Intervention, an der Leuphana Universität Lüneburg. Ihre Arbeitsschwerpunkte sind: Schulische Inklusion, Umgang mit Heterogenität in Schule und Unterricht, Evaluation schulischer Bildung.

Kontakt: poldi.kuhl@leuphana.de

Ulrike Beate Müller, Dr., ist Erziehungswissenschaftlerin und Lehrerin. Sie ist als wissenschaftliche Mitarbeiterin an der Leuphana Universität Lüneburg am Arbeitsbereich Bildungswissenschaft, insbesondere Evaluation und Intervention, tätig. Ihre Arbeitsschwerpunkte sind: Umgang mit Heterogenität in Schule und Unterricht, Verzahnung von Elementar- und Primarbereich sowie pädagogische Diagnostik.

Kontakt: ulrike_beate.mueller@leuphana.de 